\title{
Continuous Alexander-Spanier cohomology classifies principal bundles with Abelian structure group
}

\author{
by
}

\author{
B. G ünther (Frankfurt) and L. Mdzinarishvili (Tbilisi)
}

\begin{abstract}
We prove that Alexander-Spanier cohomology $H^{n}(X ; G)$ with coefficients in a topological Abelian group $G$ is isomorphic to the group of isomorphism classes of principal bundles with certain Abelian structure groups. The result holds if either $X$ is a CW-space and $G$ arbitrary or if $X$ is metrizable or compact Hausdorff and $G$ an ANR.
\end{abstract}

1. Introduction. The set $k_{G}(X)$ of isomorphism types of principal $G$ bundles over a space $X$ can be defined by means of a classifying space $k_{G}(X)=[X ; B G]$, and if the structure group $G$ is Abelian, then $B G$ can be realized as a topological Abelian group (it is the realization of a simplicial Abelian group [5, p. 87]). Therefore the construction can be iterated and we can define inductively $B^{n+1} G:=B\left(B^{n} G\right.$ ). Then $\Omega B^{n+1} G \simeq B^{n} G$ (in particular, $B G$ is an infinite loop space), so these spaces constitute an $\Omega$ spectrum and hence give rise to a generalized cohomology functor defined by $B G^{n}(X):=\left[X ; B^{n} G\right]$ for $n>0$ and by suspension in lower dimensions. The classifying spaces as path connected topological Abelian groups have trivial Postnikov invariants $\left({ }^{1}\right)$ [3, Thm. VII.3.6], therefore

$$
B G^{n}(X) \approx \prod_{m=0}^{\infty} H^{m+n}\left(X ; \pi_{m}(G)\right)
$$

for all $n \in \mathbb{Z}$ and all CW-spaces $X$. If the homotopy equivalences $\Omega B^{n+1} G \simeq$ $B^{n} G$ are appropriately chosen, then the correspondence (1) is compatible with the suspension operator, and $B G^{*}$ turns out to be a direct product of ordinary cohomology functors. One should be aware that the correspondence (1), though natural in $X$, is not natural in $G$, unless one is willing

1991 Mathematics Subject Classification: 55N05, 55R15, 55U10.

$\left({ }^{1}\right)$ Observe that the homotopy equivalence from a simplicial Abelian group to a product of Eilenberg-MacLane complexes constructed in [3, Thm. VII.3.6] is an H-group homomorphism. 
to take into account additive cohomology operations $H^{m+n}\left(-; \pi_{m}(G)\right) \rightarrow$ $H^{M+n}\left(-; \pi_{M}(H)\right)$ induced by continuous homomorphisms $G \rightarrow H$.

Here we will consider two cases: 1) The coefficient group may be an arbitrary Abelian topological group, but the class of spaces is restricted to CW-spaces, or 2) metrizable spaces or compact Hausdorff spaces are considered but the coefficient group $G$ is required to be an ANR space. In the latter case the classifying space $B G$ has the homotopy type of an ANRspace, hence $k_{G}$ and $B G^{*}$ have the continuity property familiar from Čech cohomology. Note, however, that the correspondence (1) does not carry over to this situation because direct limits do not commute with direct products. For compact Hausdorff spaces $X$ one obtains

$$
B G^{n}(X) \approx \bigoplus_{m=0}^{\infty} H^{m+n}\left(X ; \pi_{m}(G)\right)
$$

instead of (1), where the cohomology on the right hand side is Čech cohomology.

Alexander-Spanier cohomology with continuous cochains has been considered by various authors, usually with real coefficients. For arbitrary coefficient groups it was introduced by Mdzinarishvili [6]. For a space $X$ and a topological Abelian group $G$, let $C^{n}(X ; G)$ be the set of germs of mappings $X^{n+1} \rightarrow G$ defined on a neighborhood of the diagonal of $X^{n+1}$. The coboundary operator $\delta: C^{n}(X ; G) \rightarrow C^{n+1}(X ; G)$ is defined, as usual, by $\delta(\varphi)\left(x_{0}, \ldots, x_{n+1}\right)=\sum_{i=0}^{n+1}(-1)^{i} \varphi\left(x_{0}, \ldots, x_{i-1}, x_{i+1}, \ldots, x_{n+1}\right)$. The cohomology of the ensuing cochain complex is denoted by $h^{*}(X ; G)$ and is called Alexander-Spanier cohomology of $X$ with continuous coefficients in $G$ in the sense of Mdzinarishvili. The subject of this paper is to prove

$$
h^{n}(X ; G)=B H^{n-1}(X)
$$

for all $n \geq 2$ and for $X$ and $G$ as in the above mentioned two cases. In dimensions $n \leq 1, h^{n}$ violates the Eilenberg-Steenrod axioms. The group $H$ is defined as the quotient $H:=|S G| /\left|S_{0} G\right|$ of the realization of the singular complex of $G$ by the discrete subgroup of singular 0 -simplexes $\left({ }^{2}\right)$. The quotient homomorphism $|S G| \rightarrow H$ is a covering projection and allows us to identify the homotopy groups as $\pi_{m}(H)=\pi_{m}(G)$ for $m \geq 2$. To compute the fundamental group, assume that $G$ is path connected and sufficiently regular to possess a universal covering group $\widetilde{G}$. Then the composition $|S \widetilde{G}| \rightarrow$ $|S G| \rightarrow H$ is the universal covering group of $H$ and has the fiber $S_{0} \widetilde{G}$, which is algebraically nothing but $\widetilde{G}$ itself and therefore $\pi_{1}(H)=\widetilde{G}$. In particular,

$\left({ }^{2}\right)$ Group operations which are continuous only on compact subspaces are permitted. 
we get

$$
h^{2}(X ; G) \approx H^{2}(X ; \widetilde{G}) \times k_{\widetilde{G}}(X),
$$

this correspondence being natural in $X$ but not in $G$.

Locally compact Abelian groups $G$ have $\pi_{n}(G)=0$ for $n \geq 2$, so they are not too interesting for our purpose. Note, however, that for any $\mathrm{CW}$ complex $P$ the free Abelian group $F(P)$ generated by $P$ can be given a topology with $k$-continuous group law and with $\pi_{*}(F(P))=H_{*}(P ; \mathbb{Z})[1]$, whence we obtain an abundance of examples for coefficient groups.

We commence this paper with a short review of Alexander-Spanier cohomology with continuous coefficients in the sense of Mdzinarishvili. Then representing spaces for this functor will be constructed working in the simplicial category. These representing spaces turn out to be classifying spaces of certain groups, thus proving (3) for CW-spaces. The generalization to metrizable spaces follows in the next section. In the final section we consider the question under what circumstances each cocycle is cohomologous to a continuous one.

2. Alexander-Spanier cohomology with continuous coefficients. For a space $X$ and an arbitrary covering $\mathcal{A}$ we consider the cochain complex $C_{\mathcal{A}}^{*}(X ; G)$ whose $n$-cochains are continuous maps $\mathcal{A}^{n+1} \rightarrow G$, where $\mathcal{A}^{n+1} \subseteq X^{n+1}$ is the subset of $\mathcal{A}$-small $(n+1)$-tuples. In our context $X$ will be a $\mathrm{CW}$-complex or polyhedron, and $\mathcal{A}$ will be the open covering consisting of the stars of vertices of a suitable triangulation or the set of all closed simplexes. Following Mdzinarishvili [6] we define $C^{*}(X ; G)$ $:=\varliminf_{\mathcal{U}} C_{\mathcal{U}}^{*}(X ; G)$, taking the limit over all open coverings $\mathcal{U}$ of $X$, and then $h^{*}(X ; G):=H\left(C^{*}(X ; G)\right)$. We also have to consider a modified version defined by $\bar{h}^{*}(X ; G):=H\left(C_{\mathcal{A}}^{*}(X ; G)\right)$, where $\mathcal{A}$ is the closed covering of $X$ by the simplexes of a fixed triangulation. This variant reflects the combinatorial structure of $X$ more adequately and will allow the calculation of the cohomology groups in the following section. Our first task is to show that $\bar{h}^{*}$ and $h^{*}$ are equal, for which we need a few of their basic properties.

For any continuous map $f: X \rightarrow Y$ there is an obvious homomorphism $C_{\mathcal{B}}^{*}(Y ; G) \rightarrow C_{\mathcal{A}}^{*}(X ; G)$, provided $\mathcal{A}$ refines $f^{-1} \mathcal{B}$. If two maps $f, g: X \rightarrow Y$ are contiguous in the sense that for each $A \in \mathcal{A}$ there exists $B \in \mathcal{B}$ with $f(A) \cup g(A) \subseteq B$, then the induced homomorphisms are cochain homotopic (cf. [6, Def. 2.2]). From this one can derive the homotopy invariance of $h^{*}$ in full generality, but in our context this will be a byproduct of our further results.

If $X$ is represented as disjoint union $X=\coprod_{\iota} X_{\iota}$ and if $\mathcal{A}=\coprod_{\iota} \mathcal{A}_{\iota}$, then $C_{\mathcal{A}}^{*}(X ; G)=\prod_{\iota} C_{\mathcal{A}_{\iota}}^{*}\left(X_{\iota} ; G\right)$. This proves the additivity axiom (cf. $[6$, Thm. 1.2]). 
Excision properties are delicate $[6, \S 4]$. Let a polyhedron $X$ be covered by two closed subcomplexes $X=A \cup B$; we will show that their cohomology groups are related by a Mayer-Vietoris sequence that looks unusual in dimensions 0 and 1 . We denote the closed coverings of $X, A, B$ and $A \cap B=: C$ by their closed simplexes by $\mathcal{X}, \mathcal{A}, \mathcal{B}, \mathcal{C}$. Then there is an exact diagram of cochain complexes:

$$
\begin{array}{rlrlr}
0 \rightarrow C_{\mathcal{X}}^{*}(X ; G) \rightarrow C_{\mathcal{A}}^{*}(A ; G) & \times C_{\mathcal{B}}^{*}(B ; G) & & \stackrel{\alpha}{\rightarrow} & C_{\mathcal{C}}^{*}(C ; G) \\
& \downarrow \chi & & & \downarrow \chi \\
{[A ; G]} & \times[B ; G] & & \stackrel{\beta}{\rightarrow} & {[C ; G]} \\
& \downarrow & & & \downarrow \\
& 0 & & &
\end{array}
$$

Here $[C ; G]$ is the cochain complex having $[C ; G]$ as cochain group in all dimensions $\geq 0$ and alternatingly 0 and 1 as coboundary operator. Its cohomology is $[C ; G]$ in dimension 0 and zero everywhere else. The map $\chi: C_{\mathcal{C}}^{*}(C ; G) \rightarrow[C ; G]$ is given by restriction to the diagonal. The diagonal of $C^{n+1}$ is a strong deformation retract of $\mathcal{C}^{n+1}$, because a deformation can be defined by

$$
D\left(c_{0}, \ldots, c_{n} ; t\right)=\left(c_{0}^{\prime}, \ldots, c_{n}^{\prime}\right) \quad \text { with } \quad c_{i}^{\prime}=(1-t) c_{i}+\frac{t}{n+1} \sum_{j=0}^{n} c_{j} .
$$

Consequently, a cochain $\varphi \in C_{\mathcal{C}}^{n}(C ; G)$ is in the image of $\alpha$ if and only if $\chi(\varphi)$ is in the image of $\beta$, i.e. $\operatorname{im} \alpha=\chi^{-1}(\operatorname{im} \beta)$. With $\Phi^{*}:=\operatorname{im}\left\{C_{\mathcal{A}}^{*}(A ; G) \times\right.$ $\left.C_{\mathcal{B}}^{*}(B ; G) \rightarrow C_{\mathcal{C}}^{*}(C ; G)\right\}$ and $\Psi:=\operatorname{coker}\{[A ; G] \times[B ; G] \rightarrow[C ; G]\}$ (considered as a cochain complex as above) we obtain two short exact sequences of cochain complexes:

$$
\begin{aligned}
& 0 \rightarrow C_{\mathcal{X}}^{*}(X ; G) \rightarrow C_{\mathcal{A}}^{*}(A ; G) \times C_{\mathcal{B}}^{*}(B ; G) \rightarrow \Phi^{*} \rightarrow 0, \\
& 0 \rightarrow \Phi^{*} \quad \rightarrow \quad C_{\mathcal{C}}^{*}(C ; G) \quad \rightarrow \Psi \rightarrow 0 .
\end{aligned}
$$

Since $\Psi$ considered as a cochain complex has cohomology $\Psi$ in dimension 0 and zero elsewhere the second short exact sequence implies $H^{n}\left(\Phi^{*}\right)=$ $\bar{h}^{n}(C ; G)$ for $n \neq 0,1$ and for the low dimensions provides the exact sequence $0 \rightarrow H^{0}\left(\Phi^{*}\right) \rightarrow \bar{h}^{0}(C ; G) \rightarrow \Psi \rightarrow H^{1}\left(\Phi^{*}\right) \rightarrow \bar{h}^{1}(C ; G) \rightarrow 0$. Substituting this information into the first short exact sequence of cochain complexes leads to the long exact Mayer-Vietoris sequence for $\bar{h}^{*}$ in its customary form, except that $\bar{h}^{n}(C ; G)$ must be replaced by $H^{n}\left(\Phi^{*}\right)$ for $n=0,1$.

For $h^{*}$ one replaces $A$ and $B$ by neighborhoods $A^{\prime}$ and $B^{\prime}$ such that the inclusion maps $A \hookrightarrow A^{\prime}, B \hookrightarrow B^{\prime}$ and $A \cap B \hookrightarrow A^{\prime} \cap B^{\prime}$ are deformation retractions. Then the interiors of $A^{\prime}$ and $B^{\prime}$ cover $X$, and we can repeat the reasoning above to obtain a Mayer-Vietoris sequence for $h^{*}$. We have shown: 
Proposition 1. For any polyhedron $X=A \cup B$ covered by two subpolyhedra $A$ and $B$ there is a long exact Mayer-Vietoris sequence

$$
\ldots \rightarrow h^{n}(X) \rightarrow h^{n}(A) \times h^{n}(B) \rightarrow \Phi^{n} \rightarrow h^{n+1}(X) \rightarrow \ldots
$$

with $\Phi^{n}=h^{n}(A \cap B)$ for $n \geq 2$, whereas in lower dimensions we have the following exact sequnce:

$$
\begin{aligned}
0 \rightarrow \Phi^{0} \rightarrow h^{0}(A \cap B) \rightarrow \operatorname{coker}\{[A ; G] \times[B ; G] & \rightarrow[A \cap B ; G]\} \\
& \rightarrow \Phi^{1} \rightarrow h^{1}(A \cap B) \rightarrow 0 .
\end{aligned}
$$

Now let $X$ be a polyhedron with a specified triangulation, let $\mathcal{A}$ be the closed covering by its simplexes and $\mathcal{U}:=\operatorname{St}(\mathcal{V})$, where $\mathcal{V}$ is the open covering by its stars of vertices. Since $\mathcal{A}$ refines $\mathcal{U}$ there is a natural cochain map $C_{\mathcal{U}}^{*}(X ; G) \rightarrow C_{\mathcal{A}}^{*}(X ; G)$ given by restriction. If $X^{\prime}$ is the same polyhedron, but now triangulated by a subdivision, and if $\varphi: X^{\prime} \rightarrow X$ is a simplicial approximation to the identity, then the following diagram is commutative:

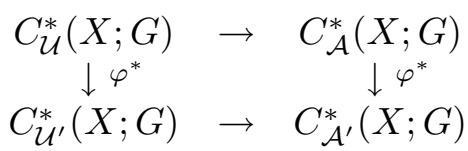

In the following section we will see that $\varphi^{*}: C_{\mathcal{A}}^{*}(X ; G) \rightarrow C_{\mathcal{A}^{\prime}}^{*}(X ; G)$ induces isomorphisms of cohomology. On the other hand, $\varphi$ is contiguous to the identity, so $\varphi^{*}: C_{\mathcal{A}}^{*}(X ; G) \rightarrow C_{\mathcal{A}^{\prime}}^{*}(X ; G)$ and $\varphi^{*}: C_{\mathcal{U}}^{*}(X ; G) \rightarrow C_{\mathcal{U}^{\prime}}^{*}(X ; G)$ are chain homotopic to the restriction map. We now observe that $\mathcal{U}$ ranges over a cofinal subset of all open coverings of $X$ if we consider all subdivisions of $X$. Hence taking direct limits of the groups $C_{\mathcal{U}}^{*}(X ; G)$ produces Mdzinarishvili's complex $C^{*}(X ; G)$ with cohomology $h^{*}(X ; G)$. The direct limit of the cochain complexes $C_{\mathcal{A}}^{*}(X ; G)$ produces a cochain complex with cohomology $\bar{h}(X ; G)$. We obtain a natural transformation $h^{*} \rightarrow \bar{h}^{*}$, which is evidently an isomorphism for one-point spaces. Since it is also compatible with the long exact Mayer-Vietoris sequences constructed above it must be an isomorphism for all polyhedra. To summarize:

Proposition 2. $h^{*}(X ; G)=\bar{h}^{*}(X ; G)$ for any polyhedron $X$ and each coefficient group $G$.

3. Classifying complexes. Let $\mathfrak{G}$ be a simplicial Abelian group; in our applications $\mathfrak{G}$ will be the singular complex of $G$. We define a sequence of simplicial Abelian groups $\mathfrak{E}(\mathfrak{G}, n)$ by $\mathfrak{E}(\mathfrak{G}, n)_{p}:=\operatorname{Hom}\left(\Delta[p]^{n+1}, \mathfrak{G}\right)$ for $n \geq$ -1 and $\mathfrak{E}(\mathfrak{G}, n)=0$ for $n<-1$, where the homomorphism $\varphi^{*}: \mathfrak{E}(\mathfrak{G}, n)_{p} \rightarrow$ $\mathfrak{E}(\mathfrak{G}, n)_{q}$ induced by $\varphi: \Delta[q] \rightarrow \Delta[p]$ is given by $\varphi^{*}:=\operatorname{Hom}\left(\varphi^{n+1}, \mathfrak{G}\right)$. $\operatorname{Hom}(\mathfrak{K}, \mathfrak{L})$ denotes the set of simplicial maps $\mathfrak{K} \rightarrow \mathfrak{L} . \Delta[p]^{0}$ is understood as a one-point complex. The 0 -skeleton $\mathfrak{E}_{0}(\mathfrak{G}, n)$ of $\mathfrak{E}(\mathfrak{G}, n)$ may be identified 
with the 0 -skeleton of $\mathfrak{G}$. We observe that $\mathfrak{E}(\mathfrak{G}, n)$ is a Kan complex $[5$, Thm. 17.1].

We denote by $p_{i}: \Delta[p]^{n+1} \rightarrow \Delta[p]^{n}$ the projection map omitting the $i$ th coordinate, $0 \leq i \leq n$, and define a simplicial homomorphism $\delta$ : $\mathfrak{E}(\mathfrak{G}, n-1) \rightarrow \mathfrak{E}(\mathfrak{G}, n)$ by $\delta:=\sum_{i=0}^{n}(-1)^{i} \operatorname{Hom}\left(p_{i}, \mathfrak{G}\right)$. This turns $\mathfrak{E}$ into a cochain complex of simplicial Abelian groups.

Lemma 3. The sequence

$$
\ldots \rightarrow \mathfrak{E}(\mathfrak{G}, n-1) \stackrel{\delta}{\rightarrow} \mathfrak{E}(\mathfrak{G}, n) \stackrel{\delta}{\rightarrow} \mathfrak{E}(\mathfrak{G}, n+1) \rightarrow \ldots
$$

is exact.

Proof. $\delta^{2}=0$ follows from $p_{j} p_{i}=p_{i} p_{j+1}$ for $j \geq i$. Now consider the simplicial maps $\varphi_{i}: \Delta[p]^{n} \rightarrow \Delta[p]^{n+1}$, which operate as identities on the $j$ th coordinates for $j \leq i$ and are 0 on the other coordinates. Then

$$
\begin{aligned}
p_{j} \varphi_{i} & = \begin{cases}\varphi_{i-1} p_{j} & \text { for } j<i, \\
\varphi_{i} p_{j-1} & \text { for } j>i+1,\end{cases} \\
p_{i} \varphi_{i} & =p_{i} \varphi_{i-1} \quad \text { for } 1<i \leq n, \\
p_{1} \varphi_{1} & =0, \quad p_{n+1} \varphi_{n}=1 .
\end{aligned}
$$

For fixed $p$ we define a homomorphism $\varrho_{n}: \mathfrak{E}(\mathfrak{G}, n+1)_{p} \rightarrow \mathfrak{E}(\mathfrak{G}, n)_{p}$ by $\varrho_{n}:=\sum_{i=1}^{n}(-1)^{i} \operatorname{Hom}\left(\varphi_{i}, \mathfrak{G}\right)$. ( $\varrho$ is not required to be a simplicial map, in fact it does not commute with the 0th boundary operator.) Then $\varrho \delta+$ $\delta \varrho=q-1$, where $q: \mathfrak{E}(\mathfrak{G}, n)_{p} \rightarrow \mathfrak{E}(\mathfrak{G}, n)_{p}$ is induced by the zero map 0 : $\Delta[p]^{n} \rightarrow \Delta[p]^{n}$. This implies that every cocycle of $\mathfrak{E}(\mathfrak{G}, n)$ is cohomologous to a cocycle in $\mathfrak{E}_{0}(\mathfrak{G}, n)$. Now observe that on the 0-skeleton the coboundary operator $\delta: \mathfrak{E}_{0}(\mathfrak{G}, n) \rightarrow \mathfrak{E}_{0}(\mathfrak{G}, n+1)$ is $\delta=0$ if $n$ is odd, $\delta=1$ if $n$ is even, and therefore its cohomology is trivial.

Lemma 4. For $n \geq 0$ the simplicial homomorphism $\alpha: \mathfrak{E}(\mathfrak{G}, n) \rightarrow \mathfrak{G}$ induced by the diagonal map $\Delta[p] \rightarrow \Delta[p]^{n+1}$ is surjective and is a homotopy equivalence.

Proof. $\alpha$ has a right inverse induced by any of the projections $\Delta[p]^{n+1}$ $\rightarrow \Delta[p]$, in particular $\alpha$ is surjective and hence [5, Lemma 18.2] a Kan fibration. Let $\mathfrak{F}(\mathfrak{G}, n) \subseteq \mathfrak{E}(\mathfrak{G}, n)$ be its fiber; we will show $\pi_{*}(\mathfrak{F}(\mathfrak{G}, n))=0$. Denote by $\Gamma \subseteq \Delta[p]^{n+1}$ the union of the diagonal and all faces $\left(d_{i} \Delta[p-1]\right)^{n+1}$, $0 \leq i \leq p$, where $d_{i}: \Delta[p-1] \rightarrow \Delta[p]$ is the $i$ th face map. Its geometric realization is a deformation retract of $\left(\Delta^{p}\right)^{n+1}$, because there is a deformation $H:\left(\Delta^{p}\right)^{n+1} \times I \rightarrow\left(\Delta^{p}\right)^{n+1}$ defined by

$$
H\left(x_{0}, \ldots, x_{n} ; t\right)=\left(y_{0}, \ldots, y_{n}\right), \quad y_{i}:=(1-t) x_{i}+\frac{t}{n+1} \sum_{j=0}^{n} x_{j},
$$


moving the whole space to the diagonal and leaving $\Gamma$ invariant. This means that $\Gamma \hookrightarrow \Delta[p]^{n+1}$ is an anodyne extension [2, Ch. IV, Def. 2.1.4]. An element of $\pi_{p}(\mathfrak{F}(\mathfrak{G}, n))$ is represented by a simplicial map $\omega: \Delta[p]^{n+1} \rightarrow \mathfrak{G}$ which is 0 on $\Gamma$, and two such maps represent the same element if they are homotopic relative $\Gamma$. Since $\Gamma \hookrightarrow \Delta[p]^{n+1}$ is an anodyne extension and $\mathfrak{G}$ a Kan complex we must have $\pi_{p}(\mathfrak{F}(\mathfrak{G}, n))=0$.

The diagram

$$
\begin{array}{cccc}
\mathfrak{E}(\mathfrak{G}, n) & & \stackrel{\alpha}{\rightarrow} & \mathfrak{G} \\
\downarrow \delta & & & \downarrow \\
\mathfrak{E}(\mathfrak{G}, n+1) & \stackrel{\alpha}{\rightarrow} & \mathfrak{G}
\end{array}
$$

is commutative if we take for the vertical map $\mathfrak{G} \rightarrow \mathfrak{G}$ the zero map for even $n$ and the identity for odd $n$, and consequently $\delta: \mathfrak{E}(\mathfrak{G}, n) \rightarrow \mathfrak{E}(\mathfrak{G}, n+1)$ is nullhomotopic, respectively a homotopy equivalence.

We set $\mathfrak{B}(\mathfrak{G}, n):=\operatorname{im}\{\delta: \mathfrak{E}(\mathfrak{G}, n-1) \rightarrow \mathfrak{E}(\mathfrak{G}, n)\}=\operatorname{ker}\{\delta: \mathfrak{E}(\mathfrak{G}, n) \rightarrow$ $\mathfrak{E}(\mathfrak{G}, n+1)\}$. Then $\delta: \mathfrak{E}(\mathfrak{G}, n) \rightarrow \mathfrak{B}(\mathfrak{G}, n+1)$ is a Kan fibration with fiber $\mathfrak{B}(\mathfrak{G}, n)$. Let $\mathfrak{F}(\mathfrak{G}, n) \subseteq \mathfrak{E}(\mathfrak{G}, n)$ be the fiber of $\alpha: \mathfrak{E}(\mathfrak{G}, n) \rightarrow \mathfrak{G}$.

Proposition 5. $\mathfrak{B}(\mathfrak{G}, n+1) \cap \mathfrak{F}(\mathfrak{G}, n+1)=B \mathfrak{B}(\mathfrak{G}, n)$ and $\mathfrak{B}(\mathfrak{G}, n+2)=$ $B(\mathfrak{B}(\mathfrak{G}, n+1) \cap \mathfrak{F}(\mathfrak{G}, n+1))$ for odd $n \geq 1$.

This proposition inductively determines these groups beginning with $\mathfrak{B}(\mathfrak{G}, 1)=\mathfrak{G} / \mathfrak{G}_{0}$

Proof. We observe $\pi_{*}(\mathfrak{F}(\mathfrak{G}, n))=0$ and consider the following exact diagram:

$$
\begin{aligned}
& 0 \rightarrow \mathfrak{F}(\mathfrak{G}, n+2) \quad \rightarrow \quad \mathfrak{E}(\mathfrak{G}, n+2) \quad \stackrel{\sim}{\rightarrow} \mathfrak{G} \quad \rightarrow \quad 0 \\
& \delta \uparrow \quad 0 \uparrow \\
& 0 \rightarrow \mathfrak{F}(\mathfrak{G}, n+1) \quad \rightarrow \quad \mathfrak{E}(\mathfrak{G}, n+1) \stackrel{\sim}{\rightarrow} \mathfrak{G} \quad \rightarrow \quad 0 \\
& 0 \rightarrow \mathfrak{F}(\mathfrak{G}, n) \quad \rightarrow \quad \mathfrak{E}(\mathfrak{G}, n) \quad \stackrel{\sim}{\rightarrow} \mathfrak{G} \rightarrow 0
\end{aligned}
$$

This implies

$$
\begin{aligned}
\mathfrak{B}(\mathfrak{G}, n) & \subseteq \mathfrak{F}(\mathfrak{G}, n), \\
\delta(\mathfrak{F}(\mathfrak{G}, n)) & =\mathfrak{B}(\mathfrak{G}, n+1) \cap \mathfrak{F}(\mathfrak{G}, n+1), \\
\mathfrak{E}(\mathfrak{G}, n+1) & =\mathfrak{F}(\mathfrak{G}, n+1)+\mathfrak{B}(\mathfrak{G}, n+1) .
\end{aligned}
$$

The last equation follows from $\alpha(\mathfrak{B}(\mathfrak{G}, n+1))=\alpha(\mathfrak{E}(\mathfrak{G}, n))=\mathfrak{G}$ and implies $\delta(\mathfrak{F}(\mathfrak{G}, n+1))=\mathfrak{B}(\mathfrak{G}, n+2)$. We obtain two fiber sequences:

$$
\begin{gathered}
0 \rightarrow \mathfrak{B}(\mathfrak{G}, n) \rightarrow \mathfrak{F}(\mathfrak{G}, n) \rightarrow \mathfrak{F}(\mathfrak{G}, n) / \mathfrak{B}(\mathfrak{G}, n) \rightarrow 0, \\
0 \rightarrow \mathfrak{B}(\mathfrak{G}, n+1) \cap \mathfrak{F}(\mathfrak{G}, n+1) \rightarrow \mathfrak{F}(\mathfrak{G}, n+1) \stackrel{\delta}{\rightarrow} \mathfrak{B}(\mathfrak{G}, n+2) \rightarrow 0 .
\end{gathered}
$$

Both bundles are classifying because their total spaces are contractible, hence $\mathfrak{F}(\mathfrak{G}, n) / \mathfrak{B}(\mathfrak{G}, n)=B \mathfrak{B}(\mathfrak{G}, n)$ and $\mathfrak{B}(\mathfrak{G}, n+2)=B \mathfrak{H}$ with $\mathfrak{H}:=$ 
$\mathfrak{B}(\mathfrak{G}, n+1) \cap \mathfrak{F}(\mathfrak{G}, n+1) \approx \mathfrak{F}(\mathfrak{G}, n) / \mathfrak{B}(\mathfrak{G}, n)$. This isomorphism is induced by $\delta: \mathfrak{F}(\mathfrak{G}, n) \rightarrow \mathfrak{B}(\mathfrak{G}, n+1) \cap \mathfrak{F}(\mathfrak{G}, n+1)$.

Definition 6. For any complex $\mathfrak{K}$ we define a cochain complex $C(\mathfrak{K} ; \mathfrak{G})^{*}$ by $C(\mathfrak{K} ; \mathfrak{G})^{n}:=\operatorname{Hom}(\mathfrak{K}, \mathfrak{E}(\mathfrak{G}, n))$ for $n \geq 0, C(\mathfrak{K} ; \mathfrak{G})^{n}=0$ for $n<0$, with coboundary operator induced by $\delta: \mathfrak{E}(\mathfrak{G}, n) \rightarrow \mathfrak{E}(\mathfrak{G}, n+1)$. Its cohomology is denoted by $h^{*}(\mathfrak{K} ; \mathfrak{G})$ and is called the cohomology of $\mathfrak{K}$ with coefficients in $\mathfrak{G}$.

Observe that the $n$-cocycles of $C(\mathfrak{K} ; \mathfrak{G})^{*}$ are simplicial maps $\mathfrak{K} \rightarrow \mathfrak{B}(\mathfrak{G}, n)$, and coboundaries are those simplicial maps that can be lifted over the Kan fibration $\delta: \mathfrak{E}(\mathfrak{G}, n-1) \rightarrow \mathfrak{B}(\mathfrak{G}, n)$. Since $\mathfrak{B}(\mathfrak{G}, n)$ is also a Kan complex the homomorphism $h^{*}(i): h^{*}(\mathfrak{L} ; \mathfrak{G}) \rightarrow h^{*}(\mathfrak{K} ; \mathfrak{G})$ induced by an anodyne extension $i: \mathfrak{K} \hookrightarrow \mathfrak{L}$ is an isomorphism, and therefore $h^{*}(\mathfrak{K} ; \mathfrak{G})$ is actually a homotopy invariant functor of the geometric realization $|\mathfrak{K}|$.

Proposition 7. For odd $n \geq 3$ there is a natural isomorphism $h^{n}(\mathfrak{K} ; \mathfrak{G})$ $\approx[\mathfrak{K} ; \mathfrak{B}(\mathfrak{G}, n)]$. For even $n \geq 2$ there is a natural isomorphism $h^{n}(\mathfrak{K} ; \mathfrak{G}) \approx$ $[\mathfrak{K} ; \mathfrak{B}(\mathfrak{G}, n) \cap \mathfrak{F}(\mathfrak{G}, n)]$.

Proof. 1) Consider $n \geq 3$ odd. We have seen that $\delta: \mathfrak{E}(\mathfrak{G}, n-1) \rightarrow$ $\mathfrak{E}(\mathfrak{G}, n)$ is nullhomotopic, and we want to show that it is also nullhomotopic when considered as a map $\delta: \mathfrak{E}(\mathfrak{G}, n-1) \rightarrow \mathfrak{B}(\mathfrak{G}, n)$. If $\beta: \mathfrak{E}(\mathfrak{G}, n-1) \rightarrow$ $\mathfrak{E}(\mathfrak{G}, n-2)$ is homotopy inverse to $\delta: \mathfrak{E}(\mathfrak{G}, n-2) \rightarrow \mathfrak{E}(\mathfrak{G}, n-1)$, then $\delta \simeq \delta^{2} \beta=0$.

By definition $h^{n}(\mathfrak{K} ; \mathfrak{G})$ is the cokernel of $\delta_{*}: \operatorname{Hom}(\mathfrak{K}, \mathfrak{E}(\mathfrak{G}, n-1)) \rightarrow$ $\operatorname{Hom}(\mathfrak{K}, \mathfrak{B}(\mathfrak{G}, n))$, and because $\delta: \mathfrak{E}(\mathfrak{G}, n-1) \rightarrow \mathfrak{B}(\mathfrak{G}, n)$ is a Kan fibration a map $\mathfrak{K} \rightarrow \mathfrak{B}(\mathfrak{G}, n)$ can be lifted to $\mathfrak{E}(\mathfrak{G}, n-1)$ if and only if it is nullhomotopic. Hence $h^{n}(\mathfrak{K} ; \mathfrak{G})=[\mathfrak{K}, \mathfrak{B}(\mathfrak{G}, n)]$.

2) Now consider $n \geq 2$ even and let $\beta_{n}: \mathfrak{G} \rightarrow \mathfrak{E}(\mathfrak{G}, n)$ be a simplicial homomorphism with $\alpha_{n} \beta_{n}=1$, and for any map $f: \mathfrak{K} \rightarrow \mathfrak{B}(\mathfrak{G}, n)$ define $f^{\prime}:=\left(1-\delta \beta_{n-1} \alpha_{n}\right) f: \mathfrak{K} \rightarrow \mathfrak{F}(\mathfrak{G}, n) \cap \mathfrak{B}(\mathfrak{G}, n)$. Observe that $\alpha_{n} \delta=\alpha_{n-1}$ because $n$ is even and therefore $\alpha_{n}\left(1-\delta \beta_{n-1} \alpha_{n}\right)=\alpha_{n}-\alpha_{n-1} \beta_{n-1} \alpha_{n}=0$. Define $h^{n}(\mathfrak{K} ; \mathfrak{G}) \rightarrow[\mathfrak{K} ; \mathfrak{B}(\mathfrak{G}, n) \cap \mathfrak{F}(\mathfrak{G}, n)]$ by $f \mapsto f^{\prime}$. This assignment is surjective because for $f(\mathfrak{K}) \subseteq \mathfrak{B}(\mathfrak{G}, n) \cap \mathfrak{F}(\mathfrak{G}, n)$ we get $f^{\prime}=f$. Now, $f$ can be lifted over $\delta: \mathfrak{E}(\mathfrak{G}, n-1) \rightarrow \mathfrak{B}(\mathfrak{G}, n)$ if and only if $f^{\prime}$ can be lifted over $\delta: \delta^{-1}(\mathfrak{F}(\mathfrak{G}, n))=\mathfrak{F}(\mathfrak{G}, n-1) \rightarrow \mathfrak{B}(\mathfrak{G}, n) \cap \mathfrak{F}(\mathfrak{G}, n)$, but since $\mathfrak{F}(\mathfrak{G}, n-1)$ is contractible this holds if and only if $f^{\prime}$ is nullhomotopic.

Proposition 8. $h^{0}(\mathfrak{K} ; \mathfrak{G})$ is the ordinary cohomology $H^{0}\left(\mathfrak{K} ; \mathfrak{G}_{0}\right)$ of $\mathfrak{K}$ with coefficients in the group of 0 -simplexes of $\mathfrak{G}$, and if $\mathfrak{G}$ is connected and $\widetilde{\mathfrak{G}}$ its universal covering group, then there is a short exact sequence

$$
0 \rightarrow H^{1}\left(\mathfrak{K} ; \pi_{1}(\mathfrak{G})\right) \rightarrow H^{1}\left(\mathfrak{K} ; \widetilde{\mathfrak{G}}_{0}\right) \rightarrow h^{1}(\mathfrak{K} ; \mathfrak{G}) \rightarrow 0 .
$$

Observe that $\pi_{1}(\mathfrak{G})$ is the fiber of $\widetilde{\mathfrak{G}} \rightarrow \mathfrak{G}$ and hence is a subgroup of $\widetilde{G}_{0}$. 
Pr o of. The equation $h^{0}(\mathfrak{K} ; \mathfrak{G})=H^{0}\left(\mathfrak{K} ; \mathfrak{G}_{0}\right)$ follows from $\mathfrak{B}(\mathfrak{G}, 0)=\mathfrak{G}_{0}$. Now assume that $\mathfrak{G}$ is connected and let $\widetilde{\mathfrak{G}}$ be the universal covering group of $\mathfrak{G}$. We observe $\widetilde{\mathfrak{G}} / \widetilde{\mathfrak{G}}_{0}=\mathfrak{G} / \mathfrak{G}_{0}=\mathfrak{B}(\mathfrak{G}, 1)$, furthermore $\mathfrak{E}(\mathfrak{G}, 0)=\mathfrak{G}$ and $\delta: \mathfrak{E}(\mathfrak{G}, 0) \rightarrow \mathfrak{B}(\mathfrak{G}, 1)$ corresponds to the quotient map $\mathfrak{G} \rightarrow \mathfrak{G} / \mathfrak{G}_{0}$. This allows us to identify $\pi_{1}(\mathfrak{B}(\mathfrak{G}, 1))$ with $\widetilde{\mathfrak{G}}_{0}$ and the image of the induced homomorphism $\delta_{\#}: \pi_{1}(\mathfrak{E}(\mathfrak{G}, 0)) \rightarrow \pi_{1}(\mathfrak{B}(\mathfrak{G}, 1))$ with $\pi_{1}(\mathfrak{G}) \subseteq \widetilde{\mathfrak{G}}_{0}$. Without loss of generality we may assume that $\mathfrak{K}$ is also connected. Since $\delta: \mathfrak{E}(\mathfrak{G}, 0) \rightarrow \mathfrak{B}(\mathfrak{G}, 1)$ is a covering projection a map $f: \mathfrak{K} \rightarrow \mathfrak{B}(\mathfrak{G}, 1)$ can be lifted over $\delta$ if and only if $\operatorname{im} f_{\#} \subseteq \pi_{1}(\mathfrak{G})$, hence the assignment $f \mapsto f_{\#}: \pi_{1}(\mathfrak{K}) \rightarrow \widetilde{\mathfrak{G}}_{0}$ embeds $h^{1}(\mathfrak{K} ; \mathfrak{G})$ into

$$
\operatorname{Hom}\left(\pi_{1}(\mathfrak{K}), \widetilde{\mathfrak{G}}_{0}\right) / \operatorname{Hom}\left(\pi_{1}(\mathfrak{K}), \pi_{1}(\mathfrak{G})\right)=H^{1}\left(\mathfrak{K} ; \widetilde{\mathfrak{G}}_{0}\right) / H^{1}\left(\mathfrak{K} ; \pi_{1}(\mathfrak{G})\right) .
$$

On the other hand, $\mathfrak{B}(\mathfrak{G}, 1)$ as a simplicial Abelian group has trivial Postnikov invariants [5, Thm. 24.5] and therefore every homomorphism $\pi_{1}(\mathfrak{K}) \rightarrow$ $\pi_{1}(\mathfrak{B}(\mathfrak{G}, 1))$ is induced by a map $\mathfrak{K} \rightarrow \mathfrak{B}(\mathfrak{G}, 1)$.

TheOREM 9. Consider a polyhedron $X$ and take for $\mathfrak{K}$ the semisimplicial complex determined by its simplexes. Let $G$ be a topological Abelian group and $\mathfrak{G}:=S(G)$ its singular complex. Then $h^{*}(\mathfrak{K} ; \mathfrak{G})$ is isomorphic to the Alexander-Spanier cohomology of $|\mathfrak{K}|=X$ with continuous coefficients in $G$ in the sense of Mdzinarishvili. The isomorphism is natural in $\mathfrak{K}$ and $\mathfrak{G}$.

Observe that in view of Propositions 5 and 7 this theorem also proves equation (3) in the introduction.

Pro of. Let $\mathcal{A}$ be the closed covering of $X$ by its simplexes and denote by $\bar{C}_{\mathcal{A}}^{*}(X ; G) \supseteq C_{\mathcal{A}}^{*}(X ; G)$ the complex of all $k$-continuous cochains $\mathcal{A}^{*+1} \rightarrow G$. The inclusion map $C_{\mathcal{A}}^{*}(X ; G) \hookrightarrow \bar{C}_{\mathcal{A}}^{*}(X ; G)$ induces a natural transformation of the corresponding cohomology functors which is an isomorphism for one-point spaces. Since the reasoning of Section 2 can be repeated for $\bar{C}_{\mathcal{A}}^{*}(X ; G)$ it has the same excision properties as $C_{\mathcal{A}}^{*}(X ; G)$ and hence our natural transformation must be an isomorphism for all polyhedra $X$. We will identify $H\left(\bar{C}_{\mathcal{A}}^{*}(X ; G)\right)$ with $\bar{h}^{*}(X ; G)$ and will show $C^{*}(\mathfrak{K} ; \mathfrak{G})=\bar{C}_{\mathcal{A}}^{*}(X, G)$ and therefore $h^{*}(\mathfrak{K} ; \mathfrak{G})=\bar{h}^{*}(X ; G)$. A cochain of $\bar{C}_{\mathcal{A}}^{n}(X, G)$ assigns to every simplex $\sigma \in \mathfrak{K}$ a map $\left(\Delta^{\operatorname{dim} \sigma}\right)^{n+1}=\left|\Delta[\operatorname{dim} \sigma]^{n+1}\right| \rightarrow G$, which by adjunction can be identified with a simplicial map $\Delta[\operatorname{dim} \sigma]^{n+1} \rightarrow S(G)$ and hence with a simplex of $\mathfrak{E}(\mathfrak{G}, n)$. In this way we obtain a 1-1 correspondence between $n$-cochains of $\bar{C}_{\mathcal{A}}^{*}(X, G)$ and simplicial maps $\mathfrak{K} \rightarrow \mathfrak{E}(\mathfrak{G}, n)$.

4. The metrizable or compact Hausdorff case. Here all spaces are assumed to be metrizable or compact Hausdorff and coefficient groups are required to be ANR-spaces.

First let us explain that the modified Mayer-Vietoris sequence introduced in Section 2 for polyhedra carries over to the present context. There 
is an exact diagram of cochain complexes similar to the one considered there:

$$
\begin{array}{rlrlrl}
0 \rightarrow C^{*}(X ; G) \rightarrow C^{*}(A ; G) & \times C^{*}(B ; G) & \stackrel{\alpha}{\rightarrow} & C^{*}(C ; G) \\
& \downarrow \chi & & & \downarrow \chi \\
{[A ; G]} & \times[B ; G] & \stackrel{\beta}{\rightarrow} & {[C ; G]} \\
& \downarrow & & & \downarrow \\
& & & & & \\
& & & & 0
\end{array}
$$

Here $A$ and $B$ are closed subspaces whose interiors cover $X$, and $C=A \cap B$. We claim that the equation $\operatorname{im} \alpha=\chi^{-1}(\operatorname{im} \beta)$ still holds and hence that the construction of the Mayer-Vietoris sequence from Section 2 can be repeated. $\operatorname{im} \alpha \subseteq \chi^{-1}(\operatorname{im} \beta)$ follows from commutativity, so let us consider a cochain $\varphi \in \chi^{-1}(\operatorname{im} \beta)$, where $\varphi$ is represented by a map $\varphi: U \rightarrow G$ defined on a neighborhood $U$ of the diagonal $\Delta_{C}$ in $C^{n+1}$. By assumption there are maps $\psi_{A}: \Delta_{A} \rightarrow G$ and $\psi_{B}: \Delta_{B} \rightarrow G$ such that $\psi_{B}-\psi_{A} \simeq \varphi$ on $\Delta_{C}$. Since $G$ is an ANR these maps may be extended over closed neighborhoods $V_{A}, V_{B}$ of the diagonals $\Delta_{A}, \Delta_{B}$ in $A^{n+1}, B^{n+1}$, respectively, and we may assume $V_{A} \cap V_{B} \subseteq U$. Taking advantage of the ANR-property once more we may, after shrinking $V_{A}$ and $V_{B}$ if necessary, extend the homotopy $\psi_{B}-\psi_{A} \simeq \varphi$ over $V_{A} \cap V_{B}$. Then $\psi_{B} \simeq \psi_{A}+\varphi$ on $V_{A} \cap V_{B}$ and $\psi_{B}$ can be replaced by a homotopic copy satisfying the strict equality $\psi_{B}=\psi_{A}+\varphi$, and these maps represent cochains $\psi_{A} \in C^{*}(A ; G)$ and $\psi_{B} \in C^{*}(B ; G)$ in the inverse image of $\varphi$ under $\alpha$. This shown, we obtain the same kind of Mayer-Vietoris sequence as in Proposition 1.

LEMma 10. The functors $h^{n}$ are pointwise taut, that is, $h^{n}\left(x_{0} ; G\right)=$ $\lim h^{n}(U ; G)$, where $U$ varies over the system of neighborhoods of a point $x_{0}$ of a metrizable space.

Proof. This is a special case of [6, Thm. 6.1], where tautness around neighborhood retracts is shown: The restriction chain map $\lim C^{*}(U ; G) \rightarrow$ $C^{*}\left(x_{0} ; G\right)$ is an epimorphism and is easily seen to be a cochain homotopy equivalence by means of the cochain homotopy

$$
\varrho(\varphi)\left(y_{0}, \ldots, y_{n-1}\right):=\sum_{i=0}^{n-1}(-1)^{i} \varphi\left(y_{0}, \ldots, y_{i}, x_{0}, \ldots, x_{0}\right) .
$$

THEOREM 11. The functors $h^{n}$ satisfy the Čech continuity property.

We observe that this means that equations (3) and (4) from the introduction carry over to the present context if Čech cohomology is used for $H^{*}$. Equation (1) does not carry over, because direct products and direct limits do not commute.

Pr o o f. We enforce continuity by taking for $\check{h}^{*}$ the Čech extension from polyhedra to metrizable spaces using limits over nerves of coverings or ANR- 
neighborhoods in suitable ambient spaces, according to one's preferences. Then $\check{h}^{*}$ satisfies the same set of axioms as $h^{*}$, including the modified MayerVietoris sequence and pointwise tautness, and there is a natural transformation of cohomology functors $\check{h}^{*} \rightarrow h^{*}$, which is an isomorphism for one-point spaces. Now our modified Mayer-Vietoris sequence is strong enough to repeat the proof of a theorem of Lawson [4, Thm. 3.2] (cf. also [7]), showing that $\check{h}^{*} \approx h^{*}$.

5. Continuous versus discontinuous cocycles. This section applies to either of the two cases specified in the introduction.

Let $G$ be a topological group and $h^{n}(X ; G) \rightarrow H^{n}(X ; G)$ the natural homomorphism induced by the inclusion of the complex of continuous Alexander-Spanier cochains into the complex of all cochains. It is our purpose to determine when this homomorphism is epimorphic. We set $\mathfrak{G}:=S(G)$. Furthermore, let $\overline{\mathfrak{G}}$ be the singular complex of $G$ with the chaotic topology. Then $\overline{\mathfrak{G}}_{0}=\mathfrak{G}_{0}, \overline{\mathfrak{G}}$ is contractible and $\overline{\mathfrak{G}} / \overline{\mathfrak{G}}_{0}=K(G, 1)$. We set $\widetilde{G}:=(G, 0)^{(I, 0)} / \Gamma$, where $\Gamma \subseteq \Omega G \subseteq(G, 0)^{(I, 0)}$ is the subgroup of nullhomotopic loops and identify the evaluation map $e_{1}: \widetilde{G} \rightarrow G$ with the homomorphism of the fundamental groups $\pi_{1}\left(\mathfrak{G} / \mathfrak{G}_{0}\right) \rightarrow \pi_{1}\left(\overline{\mathfrak{G}} / \overline{\mathfrak{G}}_{0}\right)$ induced by the inclusion map $\mathfrak{G} / \mathfrak{G}_{0} \hookrightarrow \overline{\mathfrak{G}} / \overline{\mathfrak{G}}_{0}$. If $G$ is connected, locally path-connected and semilocally 1-connected, then $e_{1}: \widetilde{G} \rightarrow G$ is the universal covering group of $G$.

Proposition 12. For every group $G$ the following two conditions are equivalent:

1. Every discontinuous Alexander-Spanier cocycle in dimension $\geq 2$ on a $C W$-complex is cohomologous to a continuos one.

2. $e_{1}: \widetilde{G} \rightarrow G$ is a split epimorphism, where the section $s: G \rightarrow \widetilde{G}$ is not required to be continuous.

These conditions are satisfied for instance if $G$ is simply connected or if $G$ is free (in the algebraic sense) and path connected.

Observe that a free Abelian group can carry non-simply connected topologies, for instance the free Abelian group generated by $S^{1}$ is a $K(\mathbb{Z}, 1)$.

Pr o of. Propositions 5 and 7 imply that the homomorphism $h^{n}(X ; G) \rightarrow$ $H^{n}(X ; G)$ may be identified with the map $\left[X, Y_{n}\right] \rightarrow[X, K(G, n)]$ induced by a certain map $Y_{n}:=B^{n-1}\left|\mathfrak{G} / \mathfrak{G}_{0}\right| \rightarrow K(G, n)$, hence 1 holds if and only if $Y_{n} \rightarrow K(G, n)$ has a right homotopy inverse. Since $Y_{n}$ is $(n-1)$-connected and has trivial Postnikov invariants such a right homotopy inverse exists if and only if the induced homomorphism $\pi_{n}\left(Y_{n}\right)=\pi_{1}\left(\mathfrak{G} / \mathfrak{G}_{0}\right) \rightarrow \pi_{1}\left(\overline{\mathfrak{G}}_{\widetilde{G}} / \overline{\mathfrak{G}}_{0}\right)=$ $\pi_{n}(K(G, n))$ splits; but this homomorphism is precisely our $e_{1}: \widetilde{G} \rightarrow G$. 


\section{References}

[1] A. Dold und R. Thom, Quasifaserungen und unendliche symmetrische Produkte, Ann. of Math. 67 (1958), 239-281.

[2] P. Gabriel and M. Zisman, Calculus of Fractions and Homotopy Theory, Ergeb. Math. Grenzgeb. 35, Springer, 1967.

[3] K. Lamotke, Semisimpliziale algebraische Topologie, Grundlehren Math. Wiss. 147, Springer, 1968.

[4] J. D. Lawson, Comparison of taut cohomologies, Aequationes Math. 9 (1973), 201209.

[5] J. P. May, Simplicial Objects in Algebraic Topology, University of Chicago Press, Midway Reprint, 1982.

[6] L. Mdzinarishvili, Partially continuous Alexander-Spanier cohomology theory, Grüne Preprintreihe der Universität Heidelberg, Heft 130, 1996.

[7] E. Michael, Local properties of topological spaces, Duke Math. J. 21 (1954), 163-171.

Fachbereich Mathematik

Johann Wolfgang Goethe-Universität

Robert-Mayer-Strasse 6-10

60054 Frankfurt, Germany

E-mail: Guenther@mathematik.uni-frankfurt.d400.de
Department of Mathematics Georgian Technical University

77 Kostava Street 380075 Tbilisi, Republic of Georgia E-mail: mdzina@imath.acnet.ge 